\title{
Testing Multi-Agent Autonomy in Complex Communications Environments
}

\author{
Lonnie T. Parker \\ Naval Undersea Warfare Center \\ Newport, RI 02841 \\ Email: lonnie.t.parker@navy.mil
}

\author{
Matthew J. Ferro \\ Naval Undersea Warfare Center \\ Newport, RI 02841 \\ Email: matthew.j.ferro@navy.mil
}

\author{
Scott R. Sideleau \\ Naval Undersea Warfare Center \\ Newport, RI 02841 \\ Email: scott.sideleau@navy.mil
}

\begin{abstract}
Advanced approaches to intelligent, real-time decision making are difficult to test for multi-agent systems. Specifically, the expense and logistics of fielding multiple unmanned underwater vehicles in an open ocean environment are significant challenges in marine autonomy. Traditionally, algorithms may be developed and tested under ideal conditions with tools that do not accurately reflect real-time performance (e.g. MATLAB). Effort is then required to integrate these algorithms onto the hardware platforms intended for in-water experimentation, so that they may be validated in a realistic setting. A simulation platform that enables the execution of complex algorithms using realistic sensor models while minimizing integration efforts enhances the ability to more productively prototype autonomy. This work highlights developments for a real-time autonomy simulator based on the common marine autonomy architecture, MOOS (MissionOriented Operating Suite), and DRI (Distributed Resource Integration), named DRI-MOOS. This unique pairing of software solutions minimizes that hardware integration requirement and expands the potential use cases for testing real-time vehicle performance and in situ decision making. This work presents example applications that use this simulator to demonstrate the impact of real-time sensor simulation for such a purpose.
\end{abstract}

Keywords-multi-agent, navigation, consensus

\section{INTRODUCTION}

The proliferation of low-cost, unmanned underwater vehicles (UUV) has increased the importance of meaningful simulation models for multi-agent scenarios. The incorporation of vehicle dynamics with sensor processing times is the next step beyond theoretical simulations and advances the state of applied robotics research. Long-term goals include updating the DRI simulation framework developed with the Distributed Control Environment (DiCE) in concert with the MOOS software to replicate realistic acoustic communication ("acomms") conditions for unmanned systems. This work presents the validation of DRI-MOOS based on tests conducted using a set of real data collected from hardware platforms during inwater experiments in Narragansett Bay, RI. To inform the DRI-MOOS system, real data was collected during separate exercises in 2015 between a host ship and a commercially available unmanned vehicle (i.e., REMUS).

The primary goal of this work is to integrate realistic representations of hardware models and environments into the simulation framework (DRI-side) so that availability of information requested by simulated agents (MOOS-side) may more accurately reflect the inherent complexities in underwater acomms. Modeling this information availability in simulation

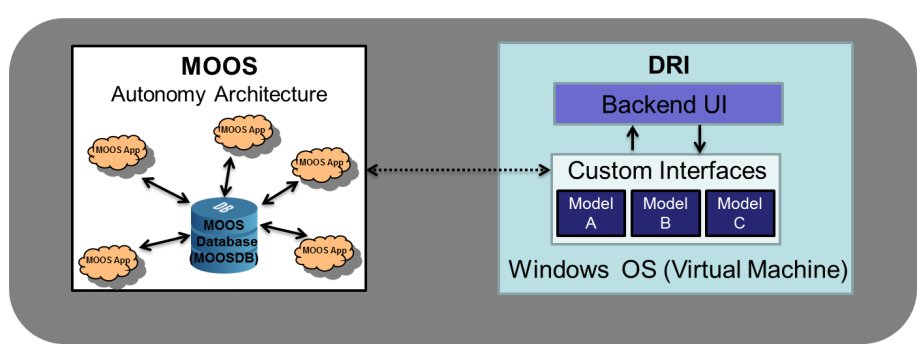

Fig. 1: DRI-MOOS architecture

amounts to replicating the uncertainty in acomms such that performance projected in simulation may be improved for inwater experiments. The objective is not to search for the best environmental model possible, since exhaustive research has already been performed in that area of study $[1,2,3]$. The objective is, instead, to explore multiple options for how the environment may be represented to the autonomous agents through simulated sensors. These options are based on real test data and provide the agents with information necessary to make navigation decisions in situ. Results will show the impact of accounting for the acoustic environment using custom modules (Figure 1).

Additional goals of testing the DRI-MOOS system include leveraging the probabilistic parameters impacting the success of communication between multiple agents in the test environment.

Experiments have been run using the DRI-MOOS simulator in a legacy state (i.e., using a range-threshold communications model) to identify any range-based trends for a two-agent acomms scenario. Following this, the in-water experiments are replicated in the simulator, allowing two vehicles to navigate in like-manner as the unmanned and host ship platforms. When the DRI model is upgraded to include user-defined environments, we repeat the experiment and illustrate the increased resolution in information provided to the user. This resolution reflects the improved performance of the rangebased and stochastic modeling of the environment.

We outline the remainder of this work as follows: in Section II, we provide an overview of DRI-MOOS and the overall architecture driving the capability to perform real-time simulation. In Section III we describe recent improvements that increase the users' options in how to represent the impact of the ocean environment on acoustic communication. In Section 
IV, we describe and show results from candidate scenarios. We conclude in Section $\mathrm{V}$ with thoughts for additional work on how this system can be improved.

\section{System ARChitecture}

The history of the DRI-MOOS simulator dates back to 2010 when a similar suite of tools was tested for a similar purpose. Researchers at the Naval Undersea Warfare Center (NUWC) collaborated with the University of Florida (UofF) and the University of Massachusetts Dartmouth (UmassD), to develop a similar system aimed at replicating real-time autonomy for multiple vehicles [4]. Duarte et al. used applications derived from the MOOS architecture to develop a framework for real-time task allocation under intermittent underwater acoustic communication.

In 2015, the authors revived this work for the purpose of testing intelligent navigation methods for multi-agent autonomy. Since vehicles can not always remain at the surface (e.g., for obtaining a continuous GPS fix), acomms must be considered as an alternative. Given the historically poor record of acomms as a reliable communication mechanism [5], understanding the strategies required to achieve multi-agent autonomy are critical to this work. Furthermore, discovering the degree of autonomous command and control (C2) that is possible under limited communication is also a focus for this research.

In our system, we rely on two inter-operable software architectures, MOOS and DRI. MOOS has been used by various academic institutions and government agencies as "middleware", enabling networked communication between supported applications. These applications adhere to a publish-subscribe architecture, where information is requested from and posted to a shared database. Particularly with respect to marine-based applications, MOOS serves a range of communities [6, 7]. The DRI software behaves as an "external" interface to the applications built in MOOS and is meant to replicate information traditionally expected from real-world environmental interaction with sensors systems. DRI, itself, is a protocol used to create models that interact with a system of environments called DiCE (Distributed Control Environment). Using shared memory, DRI models communicate with DiCE environments based on the information shared with corresponding MOOS applications in real-time (see Figure 2).

\section{APPLICATIONS, MODELS, AND ENVIRONMENTS}

The autonomy and any intelligent decision making of individual vehicles resides on the MOOS-side. MOOS provides the applications for processing information received from an acoustic modem while DRI provides the model of that modem and its operation between other modems in a simulated underwater environment. Both DRI and MOOS employ different applications and models serving unique purposes to achieve the simulated acoustic communication infrastructure desired. The modules (or "environments"), developed in DiCE, enable increased fidelity to customize the impact a simulated environment may have on the performance of the DRI sensor models with which they interact. A more detailed explanation of these sub-systems and their purpose in this application is found in Table I.
TABLE I: Description of different MOOS applications, DRI models, and DiCE environments used to construct the simulation testbed.

\begin{tabular}{c|l}
\hline \multicolumn{2}{c}{ MOOS Applications } \\
\hline pManeuver & Publishes navigation information to MOOSDB. \\
\hline iOceanServerComms & $\begin{array}{l}\text { Subscribes to navigation information from } \\
\text { MOOSDB. }\end{array}$ \\
\hline iCommsSimple & $\begin{array}{l}\text { Subscribes to communication trigger information } \\
\text { from MOOSDB and provides interface between } \\
\text { MOOS and DRI communication model, mModem. }\end{array}$ \\
\hline pSendStatus & $\begin{array}{l}\text { Publishes information to MOOSDB to "trigger" } \\
\text { periodic acomms transmission. }\end{array}$ \\
\hline mIverSimple & Control model for commercial Iver platform [8]. \\
\hline mModem & $\begin{array}{l}\text { Generic acomms model. Relays successful (or } \\
\text { failed) communications to MOOS-side from DRI. } \\
\text { DiCE Environments }\end{array}$ \\
\hline eDynamics & $\begin{array}{l}\text { Motion environment for associated vehicle models } \\
\text { and dictates successive pose. }\end{array}$ \\
\hline eAcomms & $\begin{array}{l}\text { Acomms environment for associated acomms } \\
\text { models and dictates successful/failed communica- } \\
\text { tions. }\end{array}$
\end{tabular}

When run, the user has access to the view shown in Figure 3. The two perspectives of the operational environment ("OpArea"), allow the user to visually monitor the status and make changes in real-time to vehicle behavior on the MOOSside, while maintaining an awareness of the environment's impact on the DRI-side.

\section{EXPERIMENTS AND RESULTS}

To illustrate the value of a stand-alone real-time simulation architecture, we present the following scenario:

- $\quad$ Two unmanned agents navigating according to previously defined way points.

- Defining an acomms modem model in DRI and an acomms environment in DiCE where Agent 1 periodically communicates $(0.33 \mathrm{~Hz})$ to Agent 2 .

- The events of messages successfully (and unsuccessfully) received by Agent 2 are recorded for postprocessing.

The conditions under which Agent 1 will achieve successful communication with Agent 2 is heavily dependent on the eAcomms module defined in DRI. Originally, a range-based implementation dictated whether or not a message would be transmitted between agents. A recent improvement made to eAcomms includes the ability for user-defined eAcomms models, including probabilistic models characterising specific underwater environments (e.g., shallow or deep water).

The trajectories chosen for each vehicle are drawn from actual field experiments from 2015 between a commerciallyavailable platform (REMUS 600) and a host ship with a deployed transducer to facilitate point-to-point acoustic communication. These experiments took place in Narragansett Bay with the original intent being that the REMUS autonomously navigate according to its mission goals as the host ship periodically attempted to remain in contact via acomms. Data collected from both platforms reflects their $x-y$ pose and characteristics of intermittent acoustic communication between them 4 . 


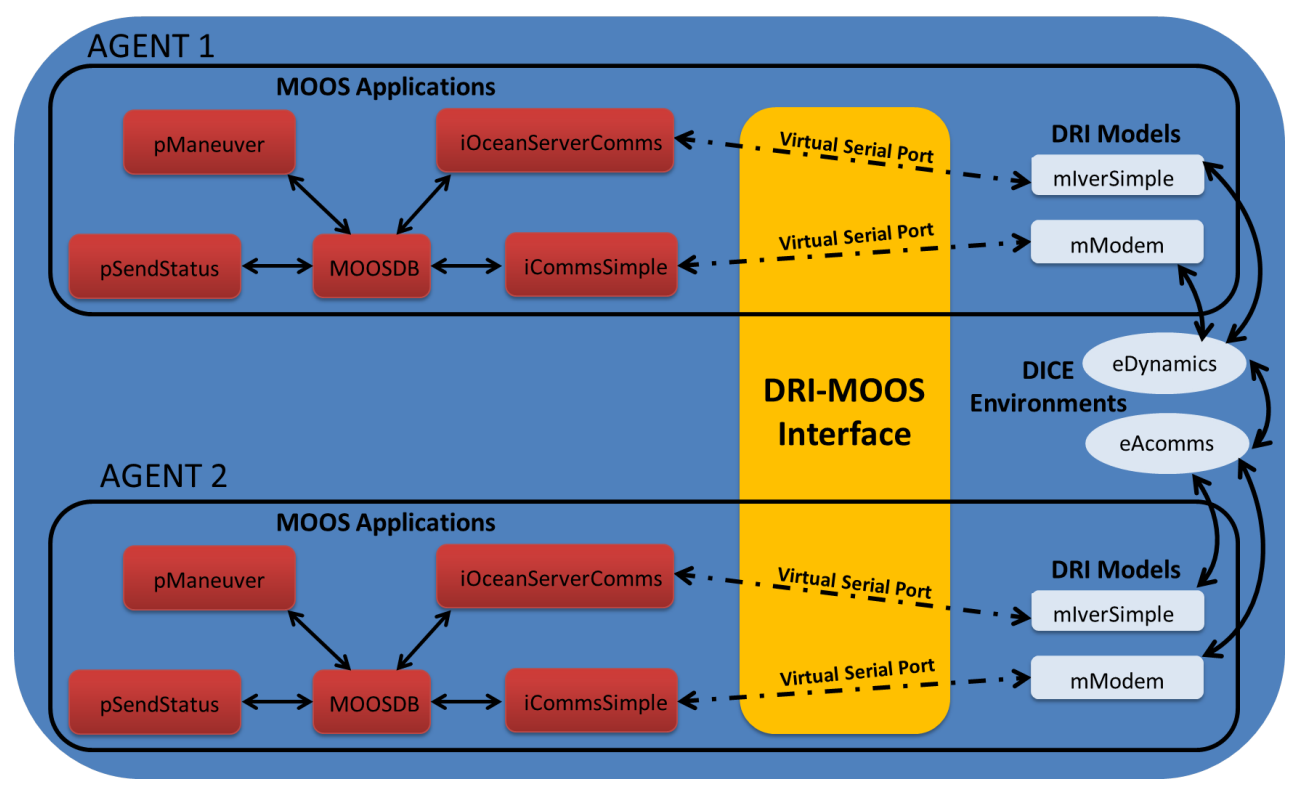

Fig. 2: DRI-MOOS architecture detailing applications and connectivity mechanisms.

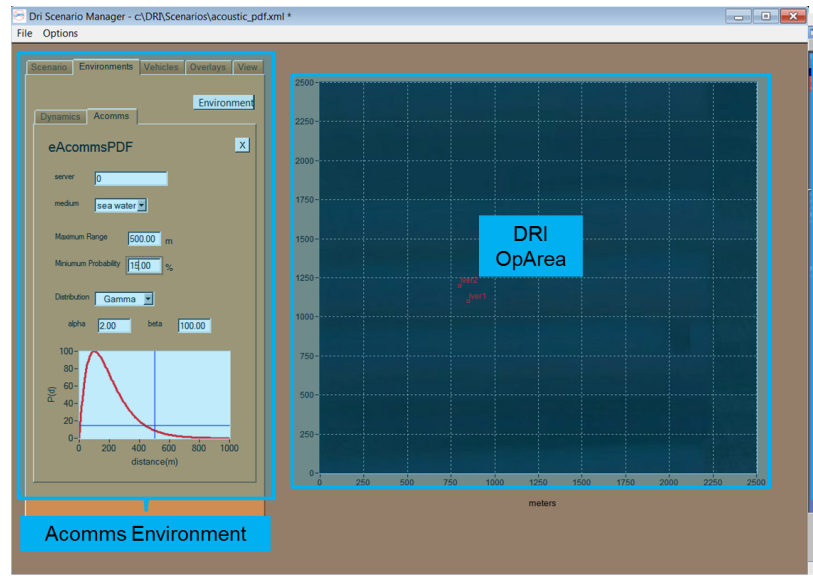

(a) DRI UI

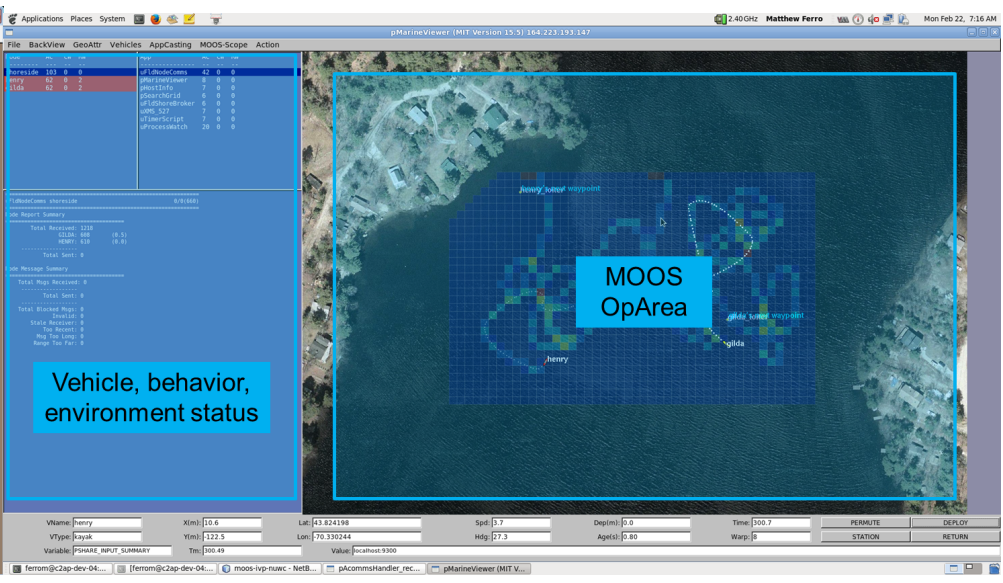

(b) MOOS UI

Fig. 3: Example DRI-MOOS display (3a, 3b) yields a location.

Using pManuever (Table I), these trajectories are fed as input to the DRI-MOOS simulator while two experiments were run, each with a different acomms environment, eAcomms. The first environment set a hard range limit defining the bounds of successful communication. For a given range, $R$, based on the separation distance, $d$, between two simulated agents (as determined by their pose information provided by mIverSimple), if $d<R$, then the eAcomms environment dictates perfect communication. While not realistic, the performance reflects the capability of the legacy model prior to the authors' improvements. While the vehicles execute the input trajectories and one periodically transmits to the other, plots of the raw data and corresponding histogram of the number of successes and failures illustrates the communication performance (see Figure 5).

Here in Figure 5, the legacy environment and models only allow for two states of communication, $100 \%$ and $0 \%$. While acomms performance can be characterized this way, it is not exhaustively accurate, as there are subtle differences in how information may be transmitted over distances and at various success rates.

The second acomms environment makes improvements upon the first by enabling the user to define a Success of Communication ( $\mathrm{SoC}$ ) curve (or function) to determine the success/failure rate of transmissions [9]. As shows in Figure $3 \mathrm{a}$, the user may select from one of several candidate $\mathrm{SoC}$ curves. Specifically, SoCs define the maximum probability of successful communication as a function of $R$. If, for example, during navigation, two vehicles are separated by $500[\mathrm{~m}]$, the maximum probability of a successful acomms transmission between them is $\operatorname{SoC}(500)$. The user may define their curve of interest to represent both known, or notional, 


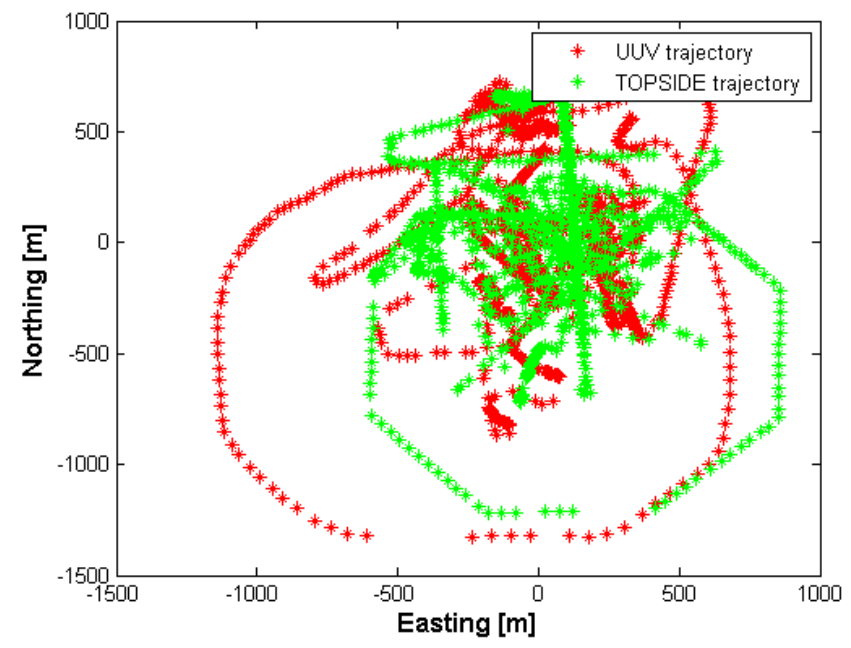

Fig. 4: Trajectories of host ship (TOPSIDE) and unmanned asset (UUV)
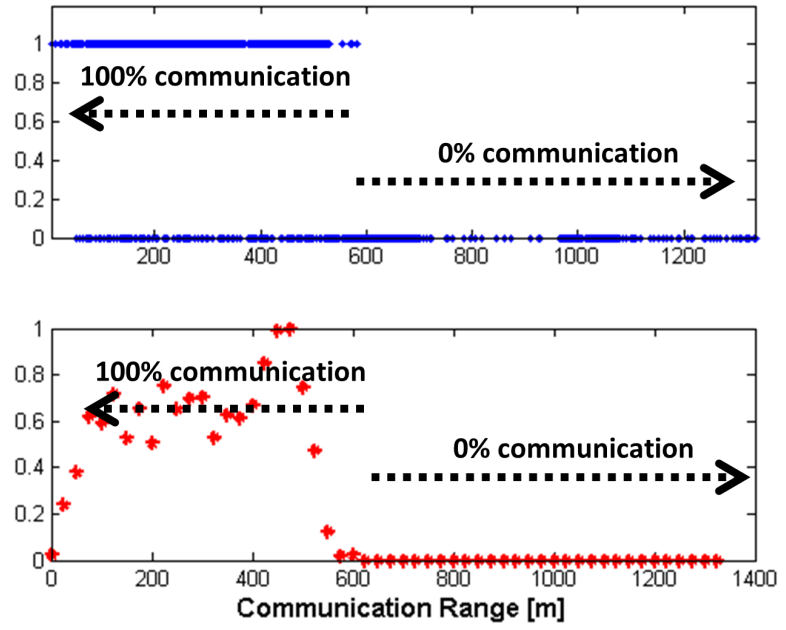

Fig. 5: Legacy eAcomms environment reflecting range-only acomms condition. Success/failure (TOP) and histogram (BOTTOM) plotted as a function of communication range.

example environments to study their impact on autonomous behaviors tested. The newer version of eAcomms retains the characteristics of the legacy version, also allowing hard range limits. As with the legacy environment, the authors evaluated the impact of periodic one-way acomms transmissions between two vehicles (see Figure 6).

Within Figure 6, it is observed that there exists more variability among the possible locations which an acomms message may be received. This increases the spectrum of possibilities for how autonomy behaviors may be designed around vehicle-to-vehicle communication. Instead of operating under the limited assumption that vehicles within a certain range of each other will always experience $100 \%$ communication, the realistic spatial and temporal advantages of how the ocean
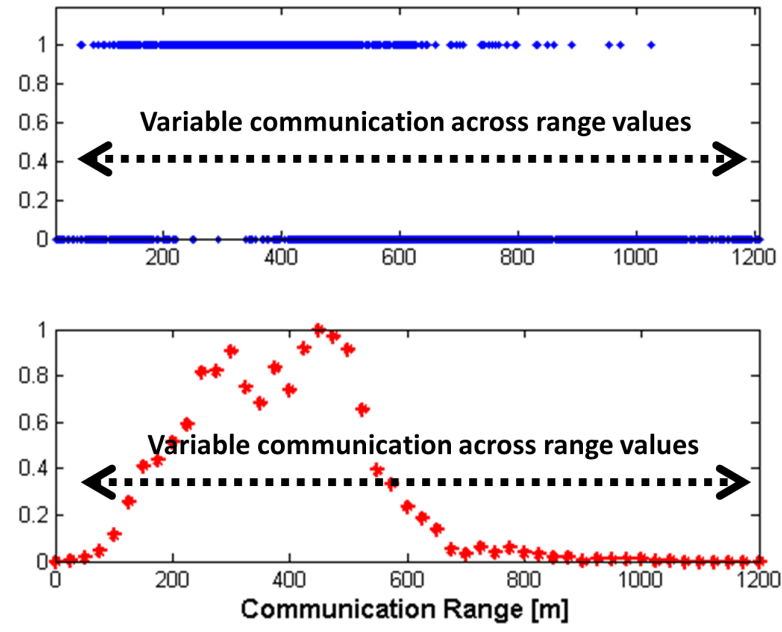

Fig. 6: Improved eAcomms environment reflecting nondeterministic acomms conditions. Success/failure (TOP) and histogram (BOTTOM) plotted as a function of communication range.

environment impacts UUV acomms can be tested.

\section{FUTURE WORK}

There are several milestones the authors expect to reach following this work. First, create a mModem model that reflects the behavior of a commercially-available system. The WHOI uModem [10], for example, is widely used in undersea communication applications, particularly maritime robotics. This achievement will increase the fidelity of the simulation, replicating expected performance from an industry standard. With respect to the eAcomms environment, range, alone, is not a sufficient enough variable on which to base the probabilistic nature of acomms performance [5]. Among others, salinity, pressure, and depth must also be incorporated into this environment and will be considered in future revisions. Next, the authors plan to incorporate Interval Programming (IvP) behaviors as a part of the DRI-MOOS infrastructure. IvP applications are commonly integrated with MOOS and increase the fidelity of autonomous behaviors that may be demonstrated in achieving scenario goals due to intermittent communication (i.e., acomms). We refer the reader to Section 2: A Very Brief Overview of MOOS in [11] for a introduction to how IvP is used with MOOS. Additionally, the authors will explore how this unique marriage of software interfaces translates to analogous success during in-water experiments. The intended "plug-and-play" design of the interfaces, both in MOOS and DRI, is such that the required integration with vehicle-specific software interfaces is minimized. These and other challenges will be explored.

\section{ACKNOWLEDGMENTS}

The authors would like to thank Ms. Christiane Duarte from the Office of Naval Research, Mr. Neil DuBois of the Chief Technology Office at the Naval Undersea Warfare Center, and 
Mr. Gerald Martel of the Naval Undersea Warfare Center, for their support of this work.

\section{REFERENCES}

[1] J. Catipovic et al., "Performance limitations in underwater acoustic telemetry," Oceanic Engineering, IEEE Journal of, vol. 15, no. 3, pp. 205-216, 1990.

[2] M. Stojanovic, "Acoustic (underwater) communications," Encyclopedia of Telecommunications, 2003.

[3] A. B. Baggeroer, "Acoustic telemetry-an overview," Oceanic Engineering, IEEE Journal of, vol. 9, no. 4, pp. 229-235, 1984.

[4] C. N. Duarte, G. R. Martel, C. M. Buzzell, D. P. Eickstedt, S. R. Sideleau, G. L. Choplin Jr., A. Johnson, C. Francolinb, A. Rao, K. DeMedeiros, and R. Balasubramanian, "Using on-board autonomous managers to support distributed cooperative autonomous systems," in MastEurope: Maritime Systems and Technology $6^{\text {th }}$ Global Conference Exhibit, Palazzo del Congressi, Rome, Italy, November 2010.

[5] I. F. Akyildiz, D. Pompili, and T. Melodia, "Underwater acoustic sensor networks: research challenges," Ad hoc networks, vol. 3, no. 3, pp. 257-279, 2005.

[6] P. Newman, "Moos::v10." [Online]. Available: https://sites.google.com/site/moossoftware/home

[7] — A MOOS-V10 Tutorial, 10th ed., Oxford University, July 2013.

[8] "Iver product information." [Online]. Available: http://www.iver-auv.com/products.html

[9] L. T. Parker, S. R. Sideleau, and B. R., "Intelligent team navigation and coordination for undersea environments," in Systems, Man, and Cybernetics (SMC), 2016. IEEE, 2016, p. to appear.

[10] WHOI Micro-Modem Software Interface Guide, Woods Hole Oceangraphic Institute, 2013.

[11] M. R. Benjamin, H. Schmidt, P. M. Newman, and J. J. Leonard, "Nested autonomy for unmanned marine vehicles with moos-ivp," Journal of Field Robotics, vol. 27, no. 6, pp. 834-875, 2010. 